\title{
Design and Research on Traffic Signal of Wireless Sensor Network Based on Labview
}

\author{
Kuangang Fan ${ }^{1,2}$ \\ ${ }^{1} \mathrm{JXUST},{ }^{2} \mathrm{CAS}$ \\ Ganzhou, China \\ kuangangfriend@163.com,
}

\author{
Jianjun Chen \\ JXUST \\ Ganzhou, China; \\ cjj530561355@163.com
}

\author{
Qingmei Cao \\ JXUST \\ Ganzhou, China \\ caoqingmeixiaocao@163.com
}

\begin{abstract}
In order to solve the increasingly serious traffic problems in the city, how to achieve effective management and control of the traffic information has become an urgent problem that should be solved in transportation of our country. This paper proposes a design on traffic signal of wireless sensor network based on LabVIEW, including traffic signal controller, GPRS wireless modem and LabVIEW interface. It adopts GPRS wireless telecommunication as the method to transport the data of traffic signal control. This method could solve the problem of high costs and the difficult problems in using wired telecommunication cable system. Traffic signal device adopts Single Chip Microcomputer to control, introduces the information collection module by using the front-end of the HMC5883L Magnetoresistive Sensor. It describes the GPRS technology and relating properties. The traffic signal monitor interface designed by LabVIEW software, and achieves the control of traffic signal.
\end{abstract}

Keywords_GPRS; Traffic Signal; HMC5883L Magnetoresistive Sensor

\section{INTRODUCTION}

Traffic is the lifeblood of city's economic. In recently years, taking advantage of advanced science and technology to improve urban traffic signal control system has become the consensus of traffic managers.

At present, installation and management of traffic light controller mainly is dedicated connection, the new management and installation. It need to excavate and string, which will cause great waste of resources unless wiring work is complete at the beginning of planning. In the management of traffic lights, using wireless sensor network (WSN) ${ }^{[1][4]}$ has the following advantages:
A. Wireless sensor nodes is small size, flexible dispenser,
less constrained and influenced by the monitored environment;
$B$. It is convenient to installation and recycling the layout of wireless sensor network;
C. Traffic information collection and road monitoring

management which based on wireless sensor network technology greatly improves the traffic condition;

D. Wireless sensor network (WSN) improves the service standard of transportation and traffic operation management.

\section{SySTEM DESIGN}

This system designs with single chip microcomputer as the core, LabVIEW interface to display, and using wireless to transfer data. According to the requirement of the design, we consider several designs about each function module. System design block diagram is shown in Figure. 1.

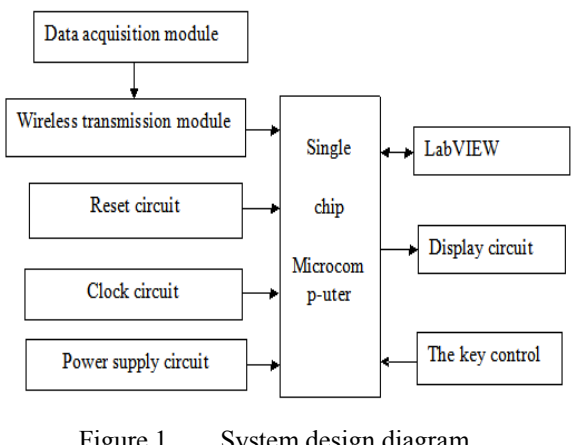

\section{COLLECTION TRAFFIC INFORMATION ALGORITHM}

The Earth's magnetic field around vehicle passes place will be disturbance. According to the characteristics of disturbance signal, dynamic vehicle detection algorithm is designed. Not only consider the differences between disturbance signals in different magnetic field background environment, but also greatly reduces the complexity of the proposed algorithm which makes it implement on the limited resources of sensor nodes. A dynamic baseline measurement method, is proposed which effectively reduces time delay and improves the acquisition precision of system. 
Suppose $\mathrm{r}(\mathrm{n})$ denotes the acquisition original signal, the magnetic field formed the dynamic measurement baseline after initialization, then detection judgment mechanism to determine, finally the state output cache will output the determination results. Whereas, if system continue stuck in a state, because of interference or other reasons, Output buffer collapses at this time, initializing the dynamic detection of baseline for the next. Specific algorithm block diagram shown in Figure. 2.

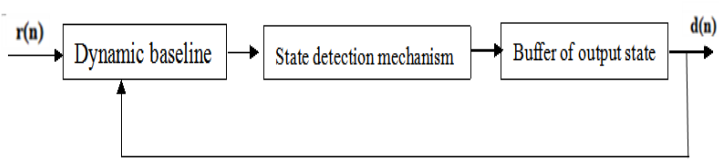

Figure 2. Dynamic vehicle

Background magnetic field intensity is different in different network deployment environment. In order to avoid the error of measurement caused by different background magnetic field strength, therefore, the concept of dynamic baseline B(n) could be introduced, the concept of magnetic sensor can be used in three coordinate axes. Assume that detection status is $S(n)$, the size of the cache for detecting state $^{S(n)}$ denotes $S_{\text {buf }}$, has the following models:

$$
B_{i}(n)=\left\{\begin{array}{c}
B_{i}(n-1) \times\left(1-\alpha_{i}\right)+r_{i}(n) \times \alpha_{i} \\
\text { iff } s(\lambda)=0 \forall \lambda \in\left[1, s_{b u f}\right] \\
B_{i}(n-1) \quad \text { others } \\
i \in[x, y, z]
\end{array}\right.
$$

Where, ${ }^{B(n)}$ denotes the dynamic baseline, $\alpha$ denotes the adjustment factor, the subscript i represents the coordinate axes. It can be seen from the formula (1) that in the absence of a vehicle by the magnetic sensor node $B(n)$ only depends on the dynamic baseline environmental background magnetic field strength, supposing $\mathrm{q}$ is 0.1 .

$$
T(n)= \begin{cases}1 & \text { iff } r_{i}(n)-B_{i}(n)>h_{i}(n) \\ -1 & \text { iff } r_{i}(n)-B_{i}(n)<-h_{i}(n) \\ 0 \quad & \text { others } \\ & i \in[x, y, z]\end{cases}
$$

Where, ${ }^{T(n)}$ denotes judgmental output exceeds the threshold under the condition of dynamic baseline, $h(n)$ denotes the threshold value.

\section{System Hardware CIRCUIT DESIGN}

\section{A. Structure and Principle of the System Circuit}

The system implement for city intersection traffic control, which composed of three major parts:

1) Information acquisition and conversion;

2) Single chip microcomputer automatic control;

3) The display.

Connection diagram of the system hardware circuit is shown in Figure.3.

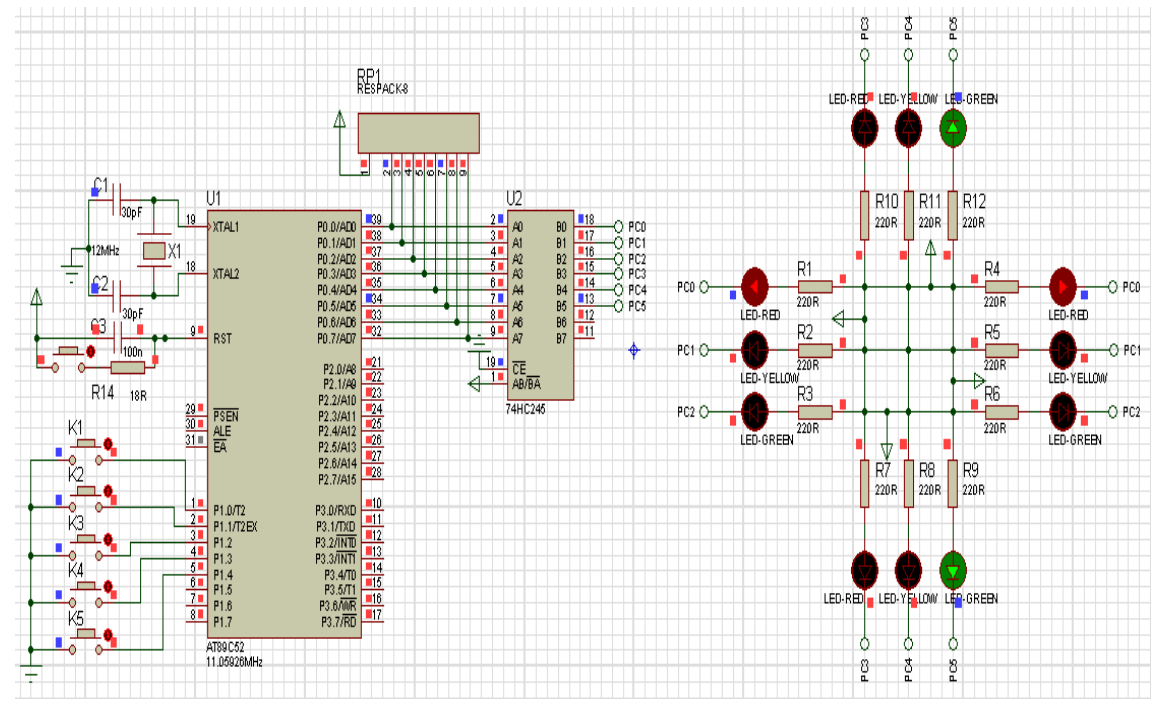

Figure 3. Connection diagram of the system hardware circuit

\section{B. SCM}

AT89C52 ${ }^{[2]}$ is a low voltage, high performance CMOS 8 bit microcontroller with that Flash program memory, can be read repeatedly wipe and read-only, 256 bytes of random access data memory (RAM) is compatible with standard MCS-51 instruction system, which built-in general 8-bit CPU and Flash storage unit.

\section{Signal Conversion Design}

Owning to sensor output signals are analog signals, while the microcontroller receives digital signal, which 
requires signal conversion circuit between the two lines, using A/D converter ADC $0809^{[5]}$.

\section{Design of Data Collection Module Circuit}

In this paper, vehicle detection based on $\mathrm{AMR}^{[6]}$ magnetic resistance sensor, it has good linear output in a certain linear region, using of this property can measure the variation of external magnetic field. Choosing Honeywell's magnetic resistance sensor HMC5883L, it is a three-axis AMR sensor, which has strong anti-jamming ability, good temperature characteristics, wide operating frequency, high-resolution, small size, easy to install, low price, etc.

\section{E. Wireless Transmission Module}

GPRS is short for General Packet Radio Service. Included in GSMPhase2.1 specification implementation, which could provide higher data rate than GSM $9.6 \mathrm{~kb} / \mathrm{s}$, the transmission rate up to $115 \mathrm{~kb} / \mathrm{s}$. Compared with the traditional circuit switching mode, GPRS introduces packet-switched transmission mode, which greatly improve the utilization rate of the wireless channel resources. Combined with the practical application environment and the requirements of the system, this paper chose to ETPro221AI GPRS module.

\section{F. LED Display Circuit Design}

According to the characteristics of this design, the display of red, yellow, green lights are indispensable, using ordinary emitting diode, setting up in each direction, a total of four groups. If the red is light in east-west direction, then green is light in the north-south direction,

On the contrary, The result is also established. Designed $\mathrm{K} 1, \mathrm{~K} 2, \mathrm{~K} 3, \mathrm{~K} 4, \mathrm{~K} 5$, a total of five keys, respectively corresponding to the five kinds of traffic patterns, which corresponding to conventional traffic, north-south traffic, east-west traffic, night traffic, no thoroughfare traffic.

\section{G. Filter}

Electric equipment is often subject to $(1000 \mathrm{HZ})$ power frequency interference, some electric equipment switch also could bring the strong electromagnetic interference. After rectifying, the DC power supply is still not ideal. It requires filter. Only a filter capacitor in parallel is needed to connect to the output of bridge rectifier. This paper designed a high-pass filter. The principle circuit design of high-pass filter is shown in Figure. 4.

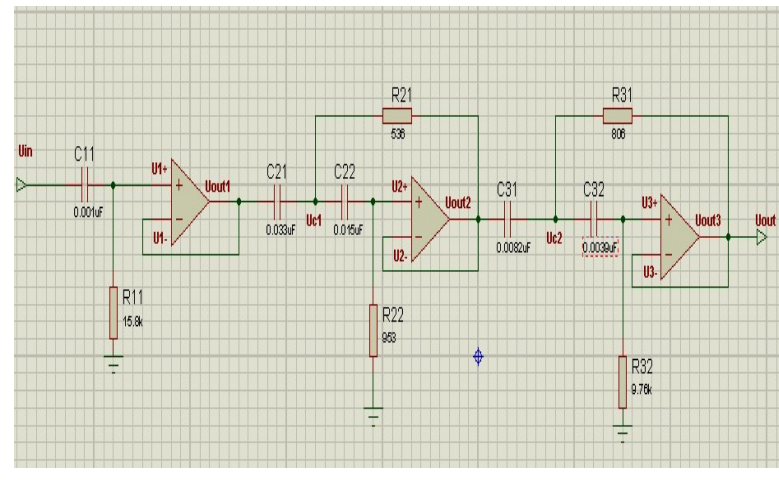

Figure 4. Principle circuit design of high-pass filter figure

Combined with all parameters of the high-pass filter circuit, it will be deduced the relationship between the input voltage and output voltage:

$$
\begin{gathered}
\frac{\text { Uout }_{1}}{\text { Uin }}=\frac{R_{11} j \omega C_{11}}{1+R_{11} j \omega C_{11}} \\
\frac{\text { Uout }_{2}}{\text { Uout }_{1}}=\frac{\left(R_{22}\right)^{2} R_{21} C_{21} C_{22} \omega^{2}}{\left(R_{22}\right)^{2} R_{21} C_{21} C_{22} \omega^{2}-j \omega R_{21} R_{22} C_{21}} \cdot \\
\frac{\text { Uout }_{3}}{\text { Uout }_{2}}=\frac{\left(R_{32}\right)^{2} R_{31} C_{31} C_{32} \omega^{2}}{\left(R_{32}\right)^{2} R_{31} C_{31} C_{32} \omega^{2}-j \omega R_{31} R_{32} C_{31}}
\end{gathered}
$$

Thus, the relationship between the input voltage and output voltage is the product of the Eq. (1)(2)(3). The parameters of equation refer to as follows: $C_{11}=0.001 \mathrm{pF}$, $R_{11}=15800 \Omega, \quad C_{21}=0.033 \mathrm{pF}, \quad C_{22}=0.015 \mathrm{pF}, \quad R_{22}=953 \Omega$, $R_{21}=536 \Omega, C_{31}=0.0002 \mathrm{pF}, C_{32}=0.0039 \mathrm{pF}, R_{31}=905 \Omega$, $R_{32}=97000 \Omega$. The stop band frequency is $1000 \mathrm{HZ}$, the pass band frequency is $10000 \mathrm{HZ}$, pass band attenuation is - $3 \mathrm{~dB}$, stop band attenuation is $-80 \mathrm{~dB}$. The simulation result is shown in Figure. 5.

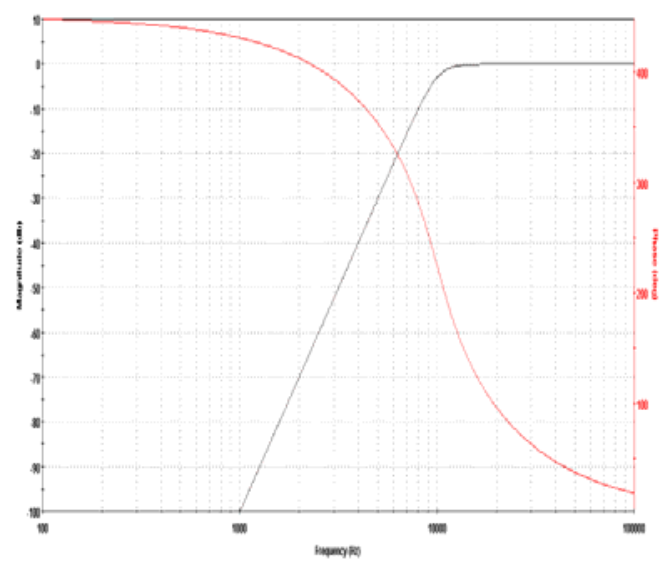

Figure 5. Simulation results of high-pass filter figure 


\section{SOFTWARE DESIGN}

In this paper, the traffic signal monitor interface is designed by LabVIEW ${ }^{[3]}$. System design of software block diagram is shown in Figure. 6.

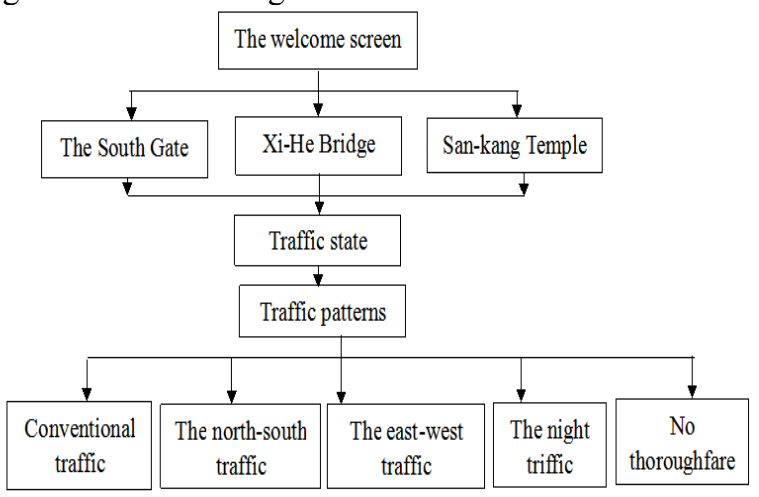

Figure.6. The system software design diagram

\section{A. The interface of Entering Window}

System interface includes enter the initial page, display product basic information. This system provides three intersection to monitoring, and clicking different button can be observed in the operation state.

\section{B. Traffic patterns}

The design of crossing traffic lights has five kinds of patterns: Conventional traffic, North-South traffic, East-West traffic, Night traffic, No Thoroughfare traffic.

Conventional traffic is described as follows: green light countdown 30 seconds and pass in the north-south direction, while no thoroughfare in east-west direction. Then yellow light in the two direction countdowns five seconds and the green light turns to red light in north-south direction, countdowns to $30 \mathrm{~s}$, while instead of the east-west direction, the cycle is continuous. The front panel of conventional pass is shown in Figure. $7^{[7]}$.

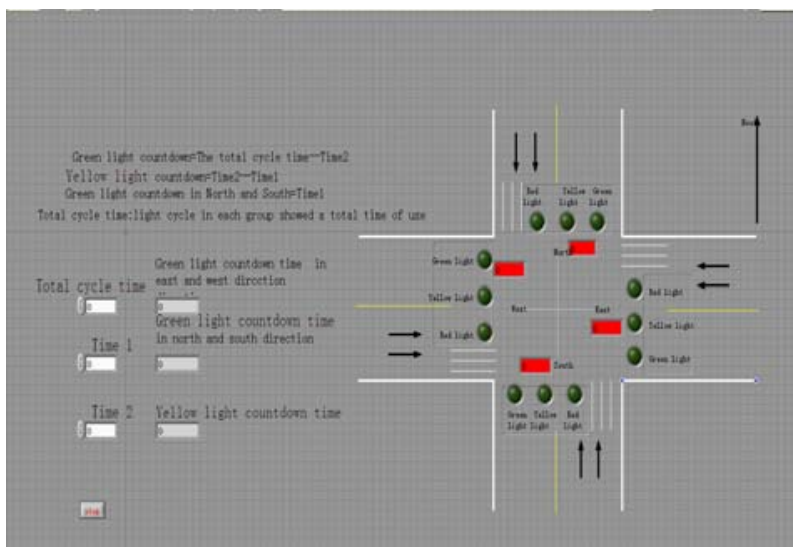

Figure 7. Conventional pass through front panel

Conventional pass program diagram is shown in Figure. 8.

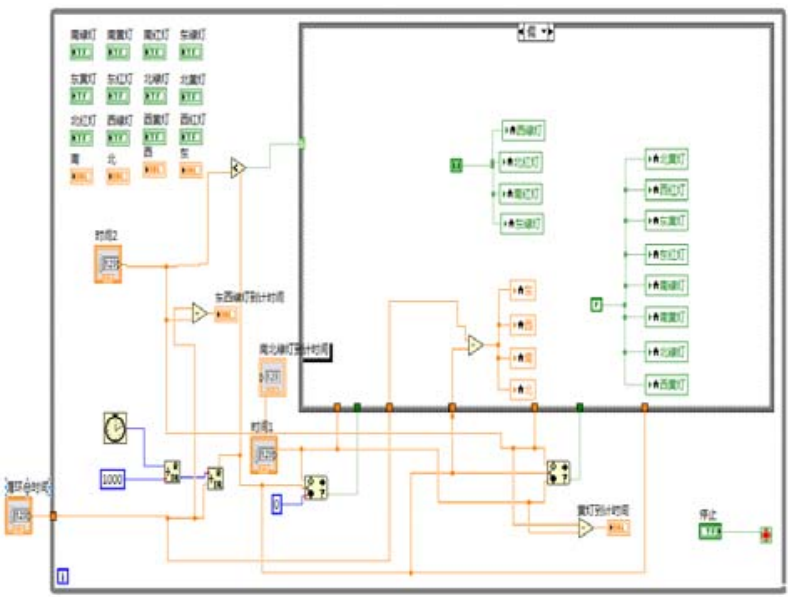

Figure 8. Conventional pass program diagram

\section{SUMMARY}

In this paper, the wireless sensor network is based on LabVIEW, which uses wireless sensor network (WSN) technology perceived as intelligent transportation front end and communication, combined with perception and fusion of GPRS technology. It provides a more convenient solution to the traffic problems. Dynamic vehicle detection algorithm is proposed, using magnetic resistance sensor for vehicle traffic caused by the geomagnetic disturbance signal. After analysis of algorithm, the system provides accurate traffic data for decision-making management of intelligent transportation in real-time.

\section{ACKNKWLEDGMENT}

The research has been supported by the Science and Technology Plan Projects of Jiangxi Provincial Education Department(GJJ12353) and the Natural Science Foundation of Jiangxi University of Science and Technology(JXXJ12046) and the National Natural Science Foundation (NO: 61163063).

\section{REFERENCES}

[1] Gong Yingying.. The Urban Traffic based on Wireless Communication [D]. Shanghai Maritime University, .2004;

[2] Sun Yaojie, GaoSai. Regional Traffic Control System based on Single Chip Microcomputer [J]. Hebei University of Technology .2012;

[3] LuPei, Liu Xiao Yong, Lu Xi. The Design of Intelligent Traffic Signal Light Control System and Simulation based on LabView [J] 2010;

[5] Li Zhaoqing. SCM Principles and Interface Technology [M]. Beijing: Beijing University of Aeronautics and Astronautics , 2010;

[6] Qi Xing Ming, Zhou Jianxing, Jiao Jin Yi,et,cl . LabVIEW8.2 Chinese Version of the Getting Started with the Typical Examples [M]. Beijing: People Post , 2010;

[7] Chen Rui. Magnetoresistive Sensor based Wireless Vehicle Inspection Measurement System Research and Implementation [D] Beijing: Beijing post Jiaotong University, Master's Thesis, 2009. 